\title{
LA UNIVERSIDAD MEXICANA EN LA SOCIEDAD DEL CONOCIMIENTO: DESAFÍOS PARA LA INCLUSIÓN SOCIAL
}

\section{THE MEXICAN UNIVERSITY IN THE KNOWLEDGE SOCIETY: CHALLENGES FOR SOCIAL INCLUSION}

\author{
Judith Cavazos Arroyo \\ Martha Leticia Gaeta González
}

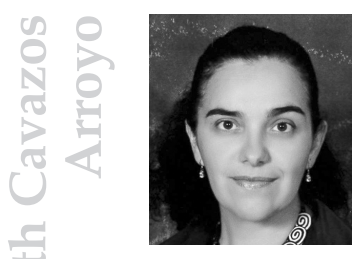

Doctorado en Dirección y Mercadotecnia; Maestría en Mercadotecnia, y Maestría en Administración, Universidad Popular Autónoma del Estado de Puebla, México. Desde 2000 es profesora investigadora en el Área de Mercadotecnia e imparte clases en el Doctorado en Pedagogía UPAEP sobre gestión educativa. Realiza su investigación en la línea de política educativa, gestión institucional y currículo, con énfasis en la educación en cultura de consumo, el marketing social y el marketing de creencias. Es miembro del Sistema Nacional de investigadores (SNI).

Correo electrónico: [judith.cavazos@upaep.mx]. 


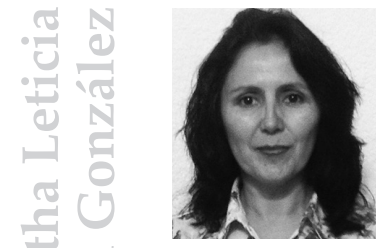

Doctorado en Psicología y Aprendizaje, Universidad de Zaragoza, España. Maestría en Psicología, Universidad de las Américas Puebla, México. Actualmente es profesora investigadora de tiempo completo del Doctorado en Pedagogía en la UPAEP. Realiza su investigación en la línea de ética, autonomía y autorregulación en los procesos de enseñanza-aprendizaje, centrando su trabajo en la autorregulación del aprendizaje y las variables educativas y psicológicas asociadas desde un enfoque contextualizado. Es miembro del Sistema Nacional de Investigadores (SNI), de la Red Mexicana de Investigadores de la Investigación Educativa (REDMIIE) y de la Red Nacional de Investigadores en Educación Valores (REDUVAL). Correo electrónico: [marthaleticia.gaeta@upaep.mx].

\section{RESUMEN}

México se ha sumado a los paradigmas que mueven a las sociedades del conocimiento, cuyo esfuerzo continuo se centra en el desarrollo tecnológico y económico, así como en la calidad de vida y la competitividad internacional. Sin embargo, sus esfuerzos no se han capitalizado en resultados sobresalientes en materia de competitividad y productividad científica, tecnológica e innovación. Tampoco se ha logrado una transformación de las universidades para generar una cultura de cohesión que contribuya a la inclusión social. Es por ello que este trabajo busca reflexionar sobre el papel y sentido de la universidad, evidenciando la realidad del país y los desafíos que deben enfrentar las universidades mexicanas ante la necesidad de promover, vía la generación, acceso y uso del conocimiento, la vinculación, participación ciudadana, equidad y cohesión social como mecanismos de inclusión social.

Palabras clave: Sociedad del conocimiento; ciencia; inclusión social; universidad; México. 


\section{ABSTRACT}

Mexico has joined the paradigms that drive knowledge societies, whose continuing effort focuses on the technological and economic development, quality of life and international competitiveness. However, its efforts has not capitalized on outstanding results in terms of competitiveness and scientific, technological and innovative productivity. Neither the country has achieved a transformation of its universities to create a culture of cohesion that could contribute to social inclusion. Therefore the main purpose of this paper is to reflect on the role and meaning of the university, showing the reality of the country and the challenges that Mexican universities have to face facing the need to promote, via the generation, access and use of knowledge, the networks, citizen participation, equity and social cohesion as mechanisms of social inclusion.

Key words: Knowledge society; science; social inclusion; university, Mexico.

\section{INTRODUCCIÓN}

La denominada Nueva Economía o economía del conocimiento, que impera en la cultura globalizada, privilegia el empleo intensivo de las tecnologías para producir nuevos conocimientos que conlleven al progreso científico y tecnológico, materializándose en innovaciones incrementales y radicales utilitarias ${ }^{1}$. La universidad no ha permanecido ajena a este proceso, insertándose en la dinámica de la competitividad global hegemónica, medida a través de la producción de conocimiento transdisciplinario, heterogéneo, en nodos y transitorio ${ }^{2}$, la creación de adelantos científicos y tecnológicos (innovación) y su eficiencia, tanto en la operación como en la realización de vinculaciones productivas de doble o triple hélice (universidad-empresa-gobierno).

1 CHAPARRO, F., «Universidad, creación de conocimiento, innovación y desarrollo», pp. 4368., en: M. Albornoz y J.A. López (eds.), Ciencia, tecnología y universidad en Iberoamérica.

2 BLEIKLIE, I. y BYRKJEFL, H., «Changing knowledge regimes: Universities in a new research environment», Higher Education, pp. 519-532. 
La generación de la dinámica ya mencionada, ha implicado que la mayor parte de las universidades hagan esfuerzos por emular los imperativos de las tendencias globales, aun cuando sus condiciones y su realidad indican que solamente pueden participar desde lo que Altbach denomina la periferia, implicando la centralización en la docencia, escasa investigación-innovación y desarticulación de las redes de globalización del conocimiento y la ciencia ${ }^{3}$. De modo que mantienen sistemas universitarios crecientemente segmentados, con capacidades y condiciones desiguales en relación con el acceso y circulación del conocimiento ${ }^{4}$. México no es la excepción y aun desde la participación periférica, la mayor parte de las universidades se han insertado en la estandarización de la calidad, participación en rankings (la mayor parte de comparación nacional), desarrollo de nuevas carreras, internacionalización y algunos productos científicos de valor utilitario incremental. En general, el recuento implica que aun incluyendo a universidades de investigación de varios países emergentes, se requieren esfuerzos onerosos, con resultados inequitativos, excluyentes y diferenciados ${ }^{5}$, al promover una educación para la competencia nacional e internacional más que para la democracia y justicia social.

Por lo anteriormente planteado, este trabajo tiene por objetivo abonar a la discusión sobre los retos y desafíos de las instituciones de educación superior mexicanas, analizando los resultados de los esfuerzos de la inserción de la universidad a la sociedad de conocimiento y las posibilidades de que estos esfuerzos funcionen como un mecanismo de inclusión social.

\section{DESARROLLO}

\section{Inserción de la universidad en la sociedad del conocimiento.-}

La esencia de la nueva economía se centra en la intensificación de estrategias de conocimiento, tanto en organizaciones lucrativas como

3 ALTBACH, P.G., «Peripheries and centres: research universities in developing countries», Higher Education Management and Policy, p. 111.

4 CARLI, S., «Conocimiento y universidad en el escenario global», pp. 146-161, en C.A. Gaytán-Riveros y J.E. Martínez-Posada (eds.), Universidad y sociedad: aproximaciones críticas, tensiones y desafíos.

5 ALTBACH, P.G., op. cit., p. 111. 
no lucrativas, para generar valor agregado y lograr una posición suficientemente competitiva a través de un conocimiento valioso, inimitable, único e insustituible, que por su alto valor y utilidad, circula a través de las redes de la globalización de la ciencia, siendo operado y comercializado bajo patentes, licencias o cualquier otro mecanismo que genere productos útiles y de alto valor económico ${ }^{6}$. En este contexto, se ha modificado de manera crucial la institución universitaria ante el gran potencial de investigación, creación y conocimiento.

Por siglos, la universidad ha apreciado la búsqueda de la verdad por sí misma, valorándose tradicionalmente la producción de ciencia pura, pero con el reciente predominio de los paradigmas del mercado en la producción de conocimiento universitario, se han acentuado las tensiones vinculadas con su autonomía, la relación utilidad-verdad y la utilidad-ética ${ }^{7}$. Además, existe una fuerte presión hacia la eficiencia de los sistemas de costos y precisión en la generación de alianzas, redes y desarrollo de proyectos, bajo el patrocinio de grandes compañías privadas para generar ciencia e innovación aplicada. Esto involucra un proceso de adaptación sobre el papel y las prácticas que deben desempeñar tanto las universidades como los centros de investigación en el mundo ${ }^{8}$.

$\mathrm{Al}$ interior de la universidad, estos cambios también permean en la reconfiguración de las competencias de docentes, profesores-investigadores, investigadores y personal administrativo, quienes deben aprender a lidiar con la complejidad que conlleva la producción de conocimiento requerida por la dinámica actual ${ }^{9}$. Como resultado, las nuevas formas de evidenciar, transparentar y comunicar las capacidades organizacionales adquiridas son la reputación institucional y las acreditaciones ${ }^{10}$; ambas funcionan como mecanismos de calidad y excelencia retomándose con ello valores económicos-empresariales.

BLEIKLIE, I. y BYRKJEFL, H., op. cit., pp. 519-532.

Cfr. Ídem.

8 CASTRO-MARTÍNEZ, E. y SUTZ, J., «Universidad, conocimiento e innovación», en M. Albornoz y J.A. López (eds.), Ciencia, tecnología y universidad en Iberoamérica.

9 RODRÍGUEZ-PONCE, E., «El rol de las universidades en la sociedad del conocimiento y en la era de la globalización: evidencia desde Chile», Interciencia, pp-822-829.

10 BLEIKLIE, I. y BYRKJEFL, H., op. cit., pp. 519-532. 


\section{Universidad e inclusión social.-}

La inclusión social en la universidad es primordialmente concebida como el acceso formal de los jóvenes a una institución de educación superior. Inclusión vinculada a equidad que involucra la redistribución de recursos, fundamentada en una ética y visión filosófica de equidad compartida y justicia social para con los miembros de una sociedad ${ }^{11}$.

Así, la universidad se contempla como un lugar de promoción de una cultura de pluralismo, de aceptación a las diferencias, de diálogo y a la vez como impulsora de la ciencia y la tecnología, con el fin de que el conocimiento produzca progreso social y equidad ${ }^{12}$, sin que por ello se conciban como procesos diferentes la educación de calidad para el progreso cultural y la justicia social.

En contextos sociales donde prevalecen fuertes asimetrías, carencia de empleo, bajos ingresos y progreso limitado es importante desarrollar políticas afirmativas que combatan la exclusión como: la cobertura, los cupos y las becas. Esto implica atender, al mismo tiempo, asuntos que faciliten el desarrollo de la inclusión como ${ }^{13}$ : autonomía, infraestructura, reforma curricular, vinculación con diferentes sectores e innovación sustentada en la generación, transmisión y aplicación del conocimiento con actores sociales y económicos del entorno.

Se considera que algunas medidas que podrían contribuir a enfrentar ciertos de los problemas de la inclusión, implican: el replanteamiento de la calidad educativa; la articulación a todos los niveles económicos y políticos del país, en materia de equidad e inclusión social; la acentuación de la importancia, desde las aulas y la cultura institucional

11 APONTE-HERNÁNDEZ, E., «Desigualdad, inclusión y equidad en la Educación Superior en América Latina y el Caribe: tendencias y escenario alternativo en el horizonte 2021», Tendencias de la Educación Superior en América Latina y el Caribe.

12 HOYOS, G., «Participación del Estado, de la comunidad académica y de la sociedad en el mejoramiento de la calidad de la Educación Superior», pp. 50-69, en C.A. Gaitán-Riveros y J.E. Martínez-Posada (eds.), Universidad y sociedad: aproximaciones críticas, tensiones y desafíos.

13 ANUIES, «Inclusión con responsabilidad social». Elementos de diagnóstico y propuestas para una nueva generación de políticas de educación superior. 
de la universidad, de la conciencia ciudadana, desde una reflexión crítica y reflexiva (esto con la finalidad de impulsar ideales y práctica de justicia social, solidaridad, paz, respeto a la diversidad, igualdad política, denuncia de injusticia, exclusión y rendición de cuentas ${ }^{14}$ a fin de operar lo que Aponte y sus colaboradores denominan el «pacto social entre los ciudadanos») ${ }^{15}$.

Específicamente en el caso mexicano, a pesar de los avances del país, aún prevalece la desigualdad en insuficiencia de opciones y oportunidades de acceso y permanencia, especialmente en los primeros años en la universidad; se evidencian la falta de diversidad de cobertura, la diversidad de condiciones de participación de oportunidades educativas y el exceso de meritocracia para el acceso ${ }^{16}$. En este sentido, la exclusión se evidencia más por ingresos, zona geográfica y etnia.

Por ello, la educación debe asumir el multiculturalismo, a través de la participación activa y la cooperación de la comunidad que permita generar una red de intereses comunes. En este sentido, las políticas de acción afirmativa se consideran mecanismos viables para reducir la exclusión social educativa, principalmente entre los alumnos que se encuentran en alto riesgo educativo, especialmente indígenas y población rural no indígena.

\section{Desempeño de México en los índices globales vinculados a la} economía del conocimiento.-

El desarrollo de ciencia, transformada en innovación y tecnología, permite lograr mejoras en el bienestar humano en múltiples áreas de la vida ${ }^{17}$. Los rankings internacionales miden estos impactos en producción científica y productividad, y competitividad económica. Los resultados más recientes sobre la región latinoamericana son clasificados como mixtos y con desafíos importantes para los próximos años ${ }^{18}$.

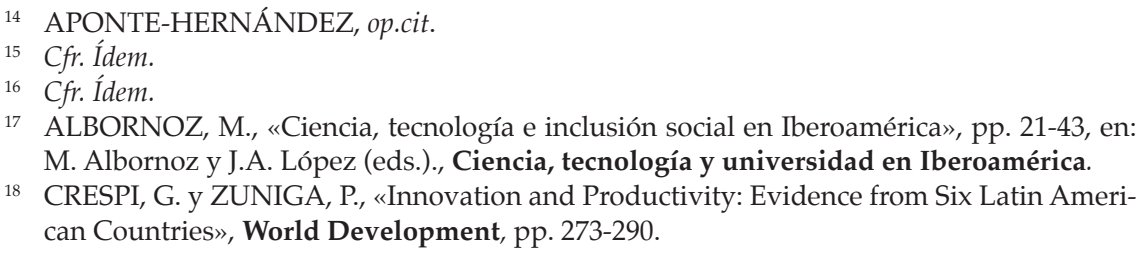


Aunque varios países de la región comparten características relativamente similares, este trabajo particulariza en el caso mexicano, pues los estilos científicos y las soluciones para la transformación de un contexto deben ser particularizados, ya que tienden a ser compatibles con el propio estilo de la sociedad.

El desempeño de México puede calificarse prácticamente en todos los índices como medio/bajo dependiendo del tema evaluado y comparativamente, dadas las condiciones de la dinámica económica y productiva del país, con otros de la propia región y otros países emergentes del globo. Diversas fuentes evidencian en México aspectos que frenan su avance científico-innovador, algunos de estos aspectos son $19,20,21,22,23,24,25,26$ :

- Debilidad estructural para que las empresas se vinculen con el sistema de innovación nacional, y para integrar los recursos científicos y tecnológicos en las estrategias de innovación.

- Entre 142 países evaluados por el índice de Innovación Global, entre 2009 y 2013, los resultados se han encontrado entre el lugar 61 y el lugar 81 (tabla 1), lo que evidencia inconsistencia en sus políticas públicas para el desarrollo innovador en los últimos cinco años.

- El Tratado de Cooperación en Materia de Patentes [PCT] (World Intellectual Property Organization [WIPO], 2013), reportó que Brasil concentró en 2012 poco más de la mitad de las aplicaciones de patentes $(53 \%)$ de la región latinoamericana, seguido por México

RICYT/OEA/CYTED, Manual de Bogotá: normalización de indicadores de innovación tecnológica en América Latina y el Caribe.

20 ZARSKY, L. Y GALLAGHER, K.P., «NAFTA, foreign direct investment, and sustainable industrial development in Mexico»., Americas Program Policy Brief, Interhemispheric Resource Center.

21 DAYTON-JOHNSON, J., Innovación en América Latina: una visión desde la perspectiva de la OCDE.

22 AECID/OEI. La nanotecnología en Iberoamérica. Situación actual y tendencias.

23 GLOBAL INNOVATION INDEX [GII]. Global Innovation Index 2013.

24 VANCE, E., «Why can't Mexico make science pay off?»., Scientific American., pp. 67-71.

25 VELÁZQUEZ, A., «Los caníbales no son verdes»., Expansión., pp. 46-47.

26 WORLD INTELLECTUAL PROPERTY ORGANIZATION [WIPO]. PTC Yearly Review. The International Patent System 2013. 
(17.1\%) (tabla 1). La mayor parte de las aplicaciones provienen de empresas, seguido de aplicaciones individuales, instituciones educativas y finalmente de gobiernos.

- Poco contacto entre los actores productivos con instituciones tecnológicas y científicas.

- Escasos o inexistentes estímulos, apoyos financieros y circulación de capital de riesgo, provenientes de financiación privada o pública para la creación de start-ups. En el país existen menos de quince fondos de capital de riesgo.

- Existe baja inversión en Investigación y Desarrollo (I+D), y más innovación de tipo organizacional y de comercialización de nuevos productos, lo que hace que la innovación sea incremental y de bajo valor en los mercados.

- Comparativamente, en publicación científica iberoamericana en nanotecnología, el país ocupa el tercer lugar, después de España y Brasil, aunque resulta mal evaluado en colaboraciones publicadas en redes iberoamericanas (lugar 11 de 14 países), concentrando principalmente sus colaboraciones con Estados Unidos.

- Asimetría entre el lugar que ocupa México como economía y su crecimiento en relación con su inversión de PIB en I+D (tabla 1). En 2013, el país ocupó la posición económica número diez a nivel mundial y según datos recientes, en 2011 invirtió el 0.43\% del PIB en I+D: menos que naciones que se encontraban en crisis el mismo año, como España $(1.33 \%)$ o Italia $(1.25 \%)$.

- Universidades y empresas productivas del país han invertido pocos esfuerzos en desarrollo científico, tecnológico y productivo en (eco) innovación y producción sustentable, prevaleciendo adopciones parciales y poco desarrolladas.

- Las pocas universidades de investigación tienen buenos científicos, pero no existen enlaces o puentes efectivos de vinculación que generen alianzas para transformar los resultados científicos en negocios operados por la industria.

- Los estímulos a los investigadores son principalmente por su antigüedad y artículos publicados, no por sus patentes o creación de laboratorios, start ups o generación de modelos de negocio innovadores, vinculados a la generación y transferencia de conocimiento. El resultado es investigación muy teórica y desvinculación con el uso y difusión del conocimiento en mercados y ciudadanos. 
- Prevalece una cultura empresarial con fuerte énfasis en la aversión al riesgo, conservadora y cuidadosa de sus inversiones, lo que provoca una dinámica de inversión-operación lenta. Aunado a ello se encuentra una burocracia exasperante para inversionistas dinámicos y una profunda fuga de cerebros. Se sabe que más del $70 \%$ de los doctores mexicanos terminan dejando el país; por ello el Consejo Nacional de Ciencia y Tecnología (CONACYT) empezó un programa de estímulos para repatriar capital intelectual vinculado a redes de investigadores en el extranjero.

\section{Índice de Innovación Global 2009-2013 (N=142)}

\begin{tabular}{|c|c|c|c|c|c|c|c|}
\hline & & & 2009 & 2010 & 2011 & 2012 & 2013 \\
\hline México & ugar 0 & do) & 61 & 69 & 81 & 79 & 63 \\
\hline \multicolumn{8}{|c|}{ Registro de Patentes 2008-2012 } \\
\hline & 2008 & 2009 & 2010 & 2011 & 2012 & $2012(\%)$ & $2011-2012(\%)$ \\
\hline \multicolumn{5}{|c|}{ Aplicaciones } & \multicolumn{2}{|c|}{ Porcentaje Regional } & Cambio comparado \\
\hline México & 203 & 194 & 191 & 225 & 190 & 17.1 & -15.6 \\
\hline \multicolumn{5}{|c|}{ Índice de Desarrollo de las TIC 2011-2012 (N=157) } & \multicolumn{3}{|c|}{ Economías Top 2013} \\
\hline & & & 2011 & 2012 & & & PPP GDO 2008-2012 \\
\hline \multicolumn{3}{|c|}{ México (lugar ocupado) } & 82 & 83 & Méxi & & $1,524,689,076,298$ \\
\hline
\end{tabular}

Fuentes: Global Innovation Index [GII], 2013; OECD, 2013; Vance, 2013; World Intellectual Property Organization [WIPO], 2013).

A partir de los planteamientos anteriores, surge la pregunta acerca de la tarea que la universidad tiene dentro de la sociedad del conocimiento, esto es, los conocimientos y valores que en ella deben promoverse para garantizar una educación de calidad e inclusión social dentro del contexto actual y futuro. 


\section{Mecanismos para la inclusión social en la universidad insertada en la} sociedad y economía del conocimiento.-

Nos hemos convertido en una sociedad que valora y adopta en medida de sus posibilidades el conocimiento y sus usos en distintas aplicaciones. Sin embargo, al mismo tiempo, vivimos una profunda decepción del mañana, acompañado del crecimiento del desempleo y la incertidumbre de que obtener un grado universitario no brinda ninguna seguridad para vivir con dignidad la vida ${ }^{27}$. El lugar central de la ciencia en la vida del siglo XXI cobra sentido en la medida que contribuye a resolver problemas vinculados a la vida humana, mejora la calidad de vida y contribuye a acercarse a la verdad. El conocimiento se ha convertido en el eje central de la inclusión-exclusión de las instituciones globales, indicando el único camino que países y regiones deberían recorrer ${ }^{28}$ para alcanzar estadios de desarrollo, crecimiento y bienestar. Así, se han creado una serie de mecanismos y estructuras que de manera sistematizada establecen jerarquías y rangos de posición y desempeño comparativo.

Las instituciones (empresas, gobiernos, universidades y centros de investigación) de los países ricos son quienes más invierten en investigación y desarrollo, produciendo resultados de innovaciones más radicales, explotando los productos científicos a través de patentes y licencias en mercados globales dependientes de ellos, por lo que mantienen su posición privilegiada. En cambio, las instituciones de países intermedios desarrollan mucha menos innovación y tecnología comparativamente con los países élite y generalmente es de tipo incremental. Principalmente estimulan la imitación o implementación de innovaciones y tecnologías extranjeras, manteniéndose una distancia muy amplia con respecto la frontera del conocimiento. Los países en fase de transición hacen grandes esfuerzos a través de la presión a sus instituciones para ser más competitivas con el fin de transitar a otra etapa, por lo que la innovación se vuelve fundamental para ello ${ }^{29}$. Los países

27 NUÑEZ, H., «Derrida, la universidad, las humanidades y la literatura»., Dossier de filosofía: destino, futuro y utopía, pp. 21-38.

28 ALBORNOZ, M., «Ciencia, tecnología e inclusión social en Iberoamérica», pp. 21-43, en: M. Albornoz y J. A. López (eds.), Ciencia, tecnología y universidad en Iberoamérica.

29 BORONDO, C., «La innovación en la literatura reciente del crecimiento endógeno», Principios, pp. 11-42. 
rezagados se vuelven totalmente dependientes del conocimiento generado y explotado por los otros países, manteniendo una situación desfavorecedora, asimétrica y excluyente en múltiples sentidos.

La universidad necesita resignificar la dicotomía inclusión/exclusión desde distintas esferas, inclusive desde el desarrollo de una cultura de cohesión científica, tecnológica e innovadora. Éste es un desafío importante ya que existen muchas tensiones para alcanzar y permear desde la comunidad universitaria el desarrollo y la difusión del conocimiento científico y tecnológico, dentro y fuera de los centros de educación superior. Así, bajo este paradigma, las universidades mexicanas suelen enfrentarse a:

- Profesores, investigadores, administrativos y estudiantes permanentemente estresados.

- Competencia generalizada por atraer a los mejores estudiantes, lo que implica que deben ser de alto rendimiento.

- Requerimientos permanentes de infraestructura para la generación de nuevos descubrimientos, inventos y desarrollos tecnológicos.

- Baja inversión en Investigación y Desarrollo (I+D).

- Incorporación institucional de una oficina de registros de inventos y patentes.

- Necesidad de desarrollo de nuevas redes de vinculación, a través de distintos mecanismos (por ejemplo, triple hélice), valorándose el contacto con centros de poder que impacten en reputación, valor organizacional y generación de co-innovaciones.

- Presencia de los investigadores de la institución en foros, congresos y publicaciones internacionales de renombre.

- Búsqueda de nuevas fuentes de financiamiento: la mayor parte de los proyectos deben ser autosustentables.

- Búsqueda de clientes para los desarrollos científicos y tecnológicos generados.

- Cultura de medición del desempeño bajo estándares internacionales, especialmente marcado por producción científica con valor comercializable, publicación en revistas en índices de prestigio global y citación de artículos publicados.

- Desarticulación de la institución con los actores del proceso de innovación. 
Propuestas recientes ${ }^{30}$ plantean una transición hacia la inclusión social a través de la cohesión social nutrida por políticas de redistribución del ingreso, equidad y ciudadanía. Desde este enfoque, el proceso educativo en la universidad tiene que ver no sólo con el desarrollo económico, sino con una transformación educativa que permita superar la pobreza, la intolerancia y la discriminación ${ }^{31}$. Caballero sostiene que estos esfuerzos, a partir de la universidad, deben realizarse desde el compromiso y la pertinencia social de la institución, a través de la creación de mecanismos de coordinación interinstitucionales para la generación de cohesión social, incluyendo a poblaciones vulnerables, desiguales y discriminadas ${ }^{32}$, lo que incorporaría al sistema de acceso a una diversidad de actores e individuos.

Asimismo, se requiere también de la construcción y el ejercicio de la ciudadanía que sustentan los derechos sociales, la libertad individual y ciudadana, el respeto a la diversidad de las identidades sociales, así como el derecho a la propiedad y la justicia, concibiendo en ello la cohesión social como un medio y un fin como marco social de reglas claras y confianza en los ámbitos social, político, económico, religioso, científico, etcétera.

\section{CONCLUSIONES}

En este trabajo hemos examinado algunos aspectos significativos sobre los retos que la inserción de la universidad mexicana en la sociedad del conocimiento tiene, en cuanto a la promoción de la calidad educativa y la repuesta a las demandas de la sociedad. Como hemos visto, los beneficios sociales se conciben como una consecuencia secundaria y natural de aquellos que genera la operación de la economía del

30 ALBORNOZ, M., "Ciencia, tecnología e inclusión social en Iberoamérica», pp. 21-43, en: M. Albornoz y J. A. López (eds.), Ciencia, tecnología y universidad en Iberoamérica.

31 HOYOS, G., «Participación del Estado, de la comunidad académica y de la sociedad en el mejoramiento de la calidad de la Educación Superior», pp. 50-69, en C. A. Gaitán-Riveros y J. E. Martínez-Posada (Eds.), Universidad y sociedad: aproximaciones críticas, tensiones y desafíos.

32 CABALLERO, J.N., «Inclusión educativa y cooperación internacional entre IES latinoamericanas y europeas como clave de cohesión social», XII Coloquio Internacional sobre Gestión Universitaria en América, pp. 1-15. 
conocimiento. Si la inclusión social es concebida como una forma ampliada de la integración, entonces es imprescindible adaptar nuestros sistemas a fin de facilitar el acceso a canales y generar el impulso de las tecnologías, especialmente de índole social.

Como se ha evidenciado, los esfuerzos de las universidades mexicanas -incluso de sus universidades de investigación - han sido insuficientes para acercarse a la denominada frontera del conocimiento e incluso para transformar sus avances científicos en utilidades científicas, tecnológicas e innovaciones que permeen transversalmente a la sociedad mexicana. Probablemente nuestro mayor desafío sea lograr una participación ciudadana más activa, coordinada, democrática, con objetivos tecno-científicos más precisos, capaz de demandar una política científica más inclusiva, que a su vez permee en la cultura y reduzca las brechas sociales con un propósito claro de mejorar la calidad de vida de los ciudadanos.

Ante esta necesidad de ampliar la oferta educativa y fortalecer la calidad, se debe plantear un sentido de calidad lo suficientemente complejo como para resistir los procesos reduccionistas, como por ejemplo, las certificaciones y las acreditaciones. Lo cual se puede lograr a partir de la búsqueda de la pertinencia, que abre a la universidad al cambio y a la innovación, con un énfasis en la atención a las necesidad sociales, tanto en relación con sus procesos de enseñanza aprendizaje, como a la investigación que en ella se realiza ${ }^{33}$, teniendo en cuenta que la universidad ha de seguir ejerciendo su vocación de «conciencia crítica» de la sociedad, de ser garante de su «humanidad» y co-creadora de una nueva sociedad $^{34}$. Tal como menciona Fierro, un pueblo educado cuenta con mejores herramientas para desarrollarse, explorar nuevas posibilidades, generar una visión integral del mundo y sus opciones ${ }^{35}$.

33 HOYOS, G., «Participación del Estado, de la comunidad académica y de la sociedad en el mejoramiento de la calidad de la Educación Superior», pp. 50-69, en C. A. Gaitán-Riveros y J. E. Martínez-Posada (Eds.), Universidad y sociedad: aproximaciones críticas, tensiones y desafíos.

34 REMOLINA, G., «¿Réquiem por la universidad?», pp. 14-21, en C. A. Gaytán-Riveros y J. E. Martínez-Posada (Eds.)., Universidad y sociedad: aproximaciones críticas, tensiones y desafíos.

35 FIERRO, J., «Difusión de la ciencia como parte de la cultura», pp. 126-137, en D. Cazés, E. Ibarra y L. Porter (Coords.), Re-conociendo a la universidad, sus transformaciones y su porvenir. 


\section{REFERENCIAS}

AECID/OEI, La nanotecnología en Iberoamérica. Situación actual y tendencias, Centro de Altos Estudios Universitarios/Organización de Estados Iberomericanos / AECID, España, 2011, 90 p.

ALBORNOZ, M., "Ciencia, tecnología e inclusión social en Iberoamérica», pp. 21-43, en: M. Albornoz y J.A. López (eds.), Ciencia, tecnología y universidad en Iberoamérica, Eudeba, Buenos Aires, 2010, 216 p.

ALTBACH, P.G., «Peripheries and centres: research universities in developing countries», Higher Education Management and Policy, vol. 19, n. 2, p. 111.

ANUIES, «Inclusión con responsabilidad social», Elementos de diagnóstico y propuestas para una nueva generación de políticas de educación superior, ANUIES, México, 2012, 70 p.

APONTE-HERNÁNDEZ, E., «Desigualdad, inclusión y equidad en la Educación Superior en América Latina y el Caribe: tendencias y escenario alternativo en el horizonte 2021», Tendencias de la Educación Superior en América Latina y el Caribe, 2008, 404 p.

BLEIKLIE, I. y BYRKJEFL, H., «Changing knowledge regimes: Universities in a new research environment», Higher Education, n. 44, pp. 519-532.

BORONDO, C., «La innovación en la literatura reciente del crecimiento endógeno», Principios, n.12, pp.11-42.

CABALLERO, J.N., «Inclusión educativa y cooperación internacional entre IES latinoamericanas y europeas como clave de cohesión social», XII Coloquio Internacional sobre Gestión Universitaria en América, pp. 1-15, en: [https://repositorio.ufsc.br/bitstream/ handle / 123456789/97754/Inclusi\% C3\% B3n\%20educativa\% 20 y\%20cooperaci\%C3\%B3n\%20internacional.pdf?sequence $=1]$. 
CARLI, S., "Conocimiento y universidad en el escenario global», pp. 146-161, en C.A. Gaytán-Riveros y J.E. Martínez-Posada (eds.), Universidad y sociedad: aproximaciones críticas, tensiones y desafíos, Colección Posgrado Pontificia Universidad Javeriana, Bogotá, 2013, 225 p.

CASTRO-MARTÍNEZ, E. y SUTZ, J., «Universidad, conocimiento e innovación», en M. Albornoz, y J.A. López (eds.), Ciencia, tecnología y universidad en Iberoamérica, Organización de Estados Ibero-Americanos, Buenos Aires, 2010, 216 p.

CHAPARRO, F., «Universidad, creación de conocimiento, innovación y desarrollo», pp. 43-68, en M. Albornoz y J.A. López (eds.), Ciencia, tecnología y universidad en Iberoamérica, Organización de Estados Ibero-Americanos, Buenos Aires, 2010, 216 p.

CRESPI, G. y ZUNIGA, P., «Innovation and Productivity: Evidence from Six Latin American Countries», World Development, n. 40, vol. 2, pp. 273-290.

DAYTON-JOHNSON, J., Innovación en América Latina: una visión desde la perspectiva de la OCDE, LATAM Innovation Index Project, 16 p.

FIERRO, J., «Difusión de la ciencia como parte de la cultura», pp. 126-137, en D. Cazés, E. Ibarra y L. Porter (coords.), Re-conociendo a la universidad, sus transformaciones y su porvenir, Tomo IV, Centro de Investigaciones Interdisciplinarias en Ciencias y Humanidades de la Universidad Nacional Autónoma de México, México, 2000, 220 p.

\section{GLOBAL INNOVATION INDEX [GII], Global Innovation Index} 2013, INSEAD, en: [http:/ / www.globalinnovationindex.org].

HOYOS, G., «Participación del Estado, de la comunidad académica y de la sociedad en el mejoramiento de la calidad de la Educación Superior», pp. 50-69, en C.A. Gaitán-Riveros y J.E. Martínez-Posada (eds.), Universidad y sociedad: aproximaciones críticas, tensiones y desafíos, Colección Posgrado Pontificia Universidad Javeriana, Bogotá, 2013, 308 p. 
NUÑEZ, H., «Derrida, la universidad, las humanidades y la literatura», Dossier de filosofía: destino, futuro y utopía, n. 75, pp.21-38.

OECD, «Expenditure on R\&D», OECD Factbook 2013: Economic, Environmental and Social Statistics, OECD Publishing, 2013, en: [http: / / dx.doi.org/10.1787/ factbook-2013-60-en].

REMOLINA, G., «¿Réquiem por la universidad?», pp. 14-21, en C.A. Gaytán-Riveros y J.E. Martínez-Posada (eds.), Universidad y sociedad: aproximaciones críticas, tensiones y desafíos, Colección Posgrado Pontificia Universidad Javeriana, Bogotá, 2013, 308 p.

RICYT/OEA/CYTED, Manual de Bogotá: normalización de indicadores de innovación tecnológica en América Latina y el Caribe, en: [http:/ / www.uis.unesco.org/Library / Documents/Bogota $\% 20$ Manual_Spa.pdf].

RODRÍGUEZ-PONCE, E., «El rol de las universidades en la sociedad del conocimiento y en la era de la globalización: evidencia desde Chile», Interciencia, n. 34, vol.11, pp-822-829.

VANCE, E., «Why can't Mexico make science pay off?», Scientific American, pp. 67-71, en: [http: / / www.scientificamerican.com/article.cfm?id=why-mexico-struggles-to-make-science-pay-off].

VELÁZQUEZ, A., «Los caníbales no son verdes», Expansión, n. 1125 , pp. 46-47.

WORLD INTELLECTUAL PROPERTY ORGANIZATION [WIPO], PTC Yearly Review. The International Patent System 2013, en: [http:/ / www.wipo.int/ipstats/en/].

ZARSKY, L. y GALLAGHER, K.P., «NAFTA, foreign direct investment, and sustainable industrial development in Mexico», Americas Program Policy Brief, Interhemispheric Resource Center, en: [http:/ / www.cipamericas.org/archives/994]. 